\title{
Nonconvex Constrained Minimisation for 3D Left Ventricular Shape Recovery Using 2D Echocardiography Data
}

\author{
Chi Young $\mathrm{Ahn}^{1}$ and Sangwoon Yun ${ }^{2, *}$ \\ ${ }^{1}$ National Institute for Mathematical Sciences, Daejeon 34047, Republic of Korea. \\ ${ }^{2}$ Sungkyunkwan University, Department of Mathematics Education, Seoul 03063, \\ Republic of Korea.
}

Received 12 March 2021; Accepted (in revised version) 22 July 2021.

\begin{abstract}
A mathematical model in the form of a nonconvex constrained minimisation problem, aimed to determine the 3D position of LV contours using 2D echocardiography data for the entire cardiac cycle is proposed. It can be considered as a quadratically constrained quadratic program in terms of one of four variables with the others fixed. The model is solved by a proximal block coordinate descent method with cyclic order and the convergence of the algorithm is proved by using the Kurdyka-Lojasiewicz property. The model does not require unsuitable assumptions in practical environments and numerical experiments show its suitability in working with real echocardiography data.
\end{abstract}

AMS subject classifications: 49M27, 68U10, 90C20, 90C26, 92C50

Key words: Nonconvex constrained minimisation, quadratically constrained quadratic program, echocardiography, left ventricle, 3D reconstruction.

\section{Introduction}

Despite the usefulness of real-time 3D echocardiography (RT3DE) [16,17], it has its limitations such as relatively high cost and poor temporal/spatial resolutions compared to 2D echocardiography. Because of that, the $2 \mathrm{D}$ echocardiography is more preferable in clinical practice. Thus most of analysis and diagnostic tools are still performed with measurements in $2 \mathrm{D}$ slices. However, it is noteworthy that recent studies on ultrafast ultrasound imaging are expected to improve the poor resolution performance of RT3DE and to provide robust analysis of the LV behavior $[3,6]$.

This work is the continuation of our studies $[1,2]$ on the 3D left ventricle (LV) border recovery from 2D echocardiography data. This topic attracts substantial interest because the dynamic 3D motion of the heart is observed without using 3D imaging scanners. The main

${ }^{*}$ Corresponding author. Email addresses: chiyoung@nims.re.kr (C. Y. Ahn), yswmathedu@skku.edu (S. Yun) 
problem consists in obtaining image data of multiple images and making an interpolation of the cardiac chambers in 3D. Thus the 3D representation of the $\mathrm{LV}$ shape requires the 3D position information of multiple 2D imaging planes. There are various studies on recovering 3D LV shape from 2D echocardiography - cf. [9,11,19], where additional devices, such as motor or position sensors are used for rotating 2D imaging probe mechanically or by sensing the probe positions. The 3D position and orientation of 2D image planes are spatially pre-determined or tracked using a sensor attached to a probe [15].

Similar to $[1,2]$, this paper focuses on the problem of establishing the 3D position of imaging planes associated with multiple 2D apical echocardiograms, which are obtained with involvement of any additional device. In particular, previously we considered mathematical models based on the fact that the angles between apical long-axis 4, 2 and 3chamber views are approximately $60^{\circ}$ toward the each other [14], cf. Fig. 1. It was also assumed that the imaging planes corresponding to the three views intersect at the same axis and the circumferential length of mitral annulus with small variation throughout the whole cardiac cycle is in the fixed range. Under such assumptions, the models were applied to still image data.

The aim of this work is to build a new 3D recovery model without the assumptions used in the previous models. Those assumptions are removed by using moving image data acquired during the whole cardiac cycle. The only assumption is that an imaging probe is fixed without any movement for any heartbeat period smaller than 1 second. This means that throughout the entire cardiac cycle, the apical long-axis 2-, 3- and 4-chamber views are fixed and the motion of LV borders is observed only on fixed planes. It uses $2 \mathrm{D}$ echocardiography data obtained during the entire cardiac cycle. As shown in Fig. 3, it is expected that the distance between the LV control points moving in every fixed plane holds a proper distance in space. Wrong positions of the planes may lead to a very irregular and large distance in space between the control points. Hence, we propose a model maintaining

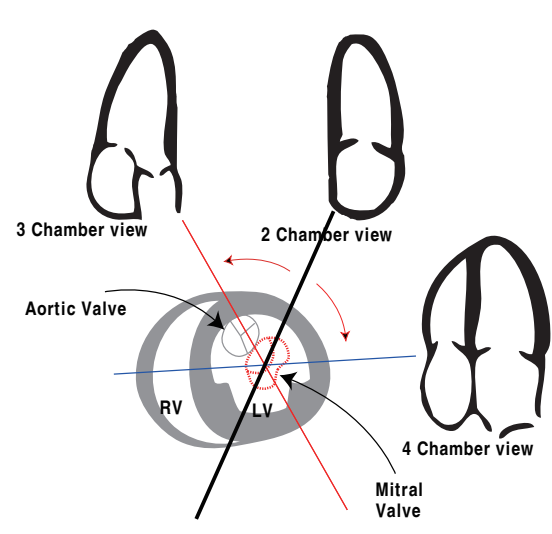

(a)

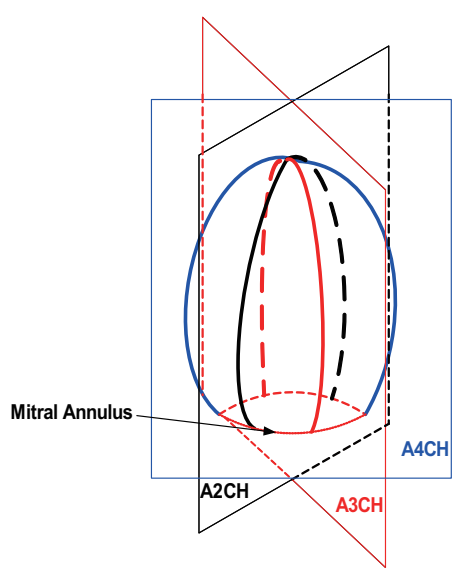

(b)

Figure 1: Apical 4-, 2- and 3-chamber views of cardiac images. The images are obtained by clockwise and counterclockwise rotating of the scanning probe by $60^{\circ}$. 


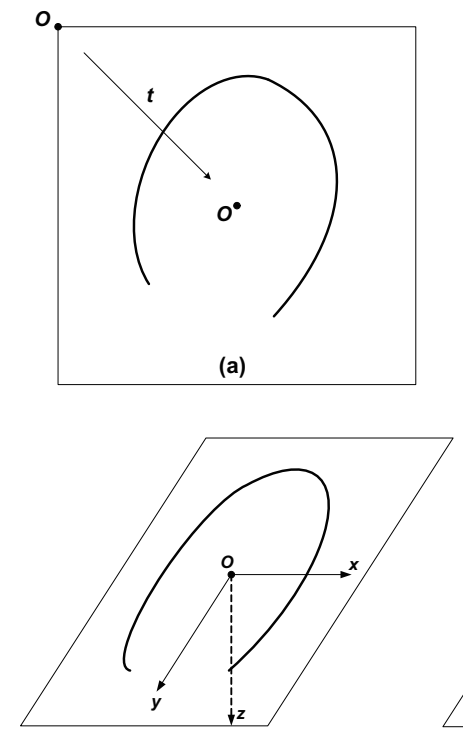

(c)

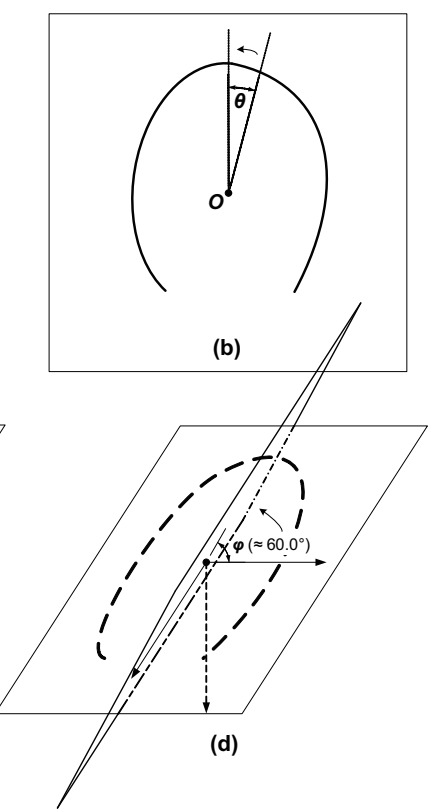

Figure 2: Transformation of 2D LV contours into 3D ones by translation and rotation angles $\theta$ and $\varphi$.

a proper distance between the control points in space. The proposed model is a nonconvex constrained minimisation problem challenging to solve. However, it is a quadratically constrained quadratic program (QCQP) [10] in terms of one of four variables with the others fixed. We exploit this structure to introduce a proximal block coordinate descent method with cyclic order (PBCDC) using OPTI solver [12] for QCQP - i.e. we cyclically minimise the proximal form [18] of the model with respect to one of the variables with the others fixed by using the QCQP solver. The objective function of the proposed model is a semialgebraic function and, hence, is a Kurdyka-Lojasiewicz function [5]. Taking into account the Kurdyka-Lojasiewicz property, we show that the whole sequence of the algorithm converges to a stationary point. We carry out numerical experiments to show that the model can efficiently work with real echocardiography data.
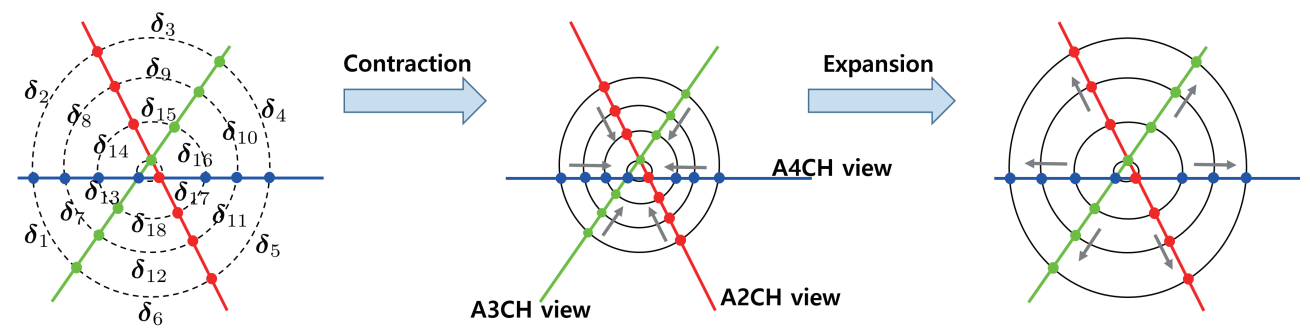

Figure 3: Contraction and dilation of LV during heartbeat. Three fixed imaging planes viewed from apex (RGB lines), circumference along LV boundary (dashed line) and LV control points (RGB dots) moving on the planes during heartbeat. 


\section{Mathematical Model for Computing 3D Positions}

Here we formulate the mathematical model for finding the 3D position of the image planes in the form of a nonconvex constrained minimisation problem. The apical long-axis 2, 3 and 4-chamber views are abbreviated as $\mathrm{A} 2 \mathrm{CH}, \mathrm{A} 3 \mathrm{CH}$ and $\mathrm{A} 4 \mathrm{CH}$, respectively. The solution of the model is obtained by the proximal block coordinate descent method with cyclic order, the convergence of which is proved.

\subsection{A model formulation}

We start with the estimate of the 3D positions corresponding to the 2D LV contours. Let $\Omega \subseteq \mathbb{R}^{2} \times\{0\}$ be an imaging domain - i.e. $\Omega:=[0,255] \times[0,255] \times\{0\}$. The LV contour in an ultrasound image is denoted by a parametric contour $\mathscr{C}=\{\mathbf{p}(s)=(x(s), y(s), 0) \mid 0 \leq$ $s \leq 1\}$, which can be identified as its $n$ contour points $\mathbf{p}_{1}=\mathbf{p}\left(s_{1}\right), \ldots, \mathbf{p}_{n}=\mathbf{p}\left(s_{n}\right)$, where $0=s_{1}<s_{2}<\cdots<s_{n}=1$. The sets of LV contour points for the A4CH, A3CH and A2Ch views are defined by $\mathscr{C}_{p}=\left\{\mathbf{p}_{i} \in \Omega: i=1, \ldots, n\right\}, \mathscr{C}_{q}=\left\{\mathbf{q}_{i} \in \Omega: i=1, \ldots, n\right\}$ and $\mathscr{C}_{r}=\left\{\mathbf{r}_{i} \in \Omega: i=1, \ldots, n\right\}$, respectively. We also denote the unknown spatial translation variables for $\mathscr{C}_{p}$ and $\mathscr{C}_{q}$ by $\boldsymbol{\alpha}$ and $\boldsymbol{\beta} \in[-255,255]^{3} \subseteq \mathbb{R}^{3}$, respectively. In addition, $\theta$ is the angle to rotate counterclockwise around the translated origin and $\varphi$ is the angle to rotate around $y$-axis in a 2D ultrasound image to represent the LV contour in a 3D spatial domain - cf. Fig. 2.

Let $\mathbf{s}_{i}$ and $\mathbf{t}_{i}$ be the geometrically transformed 3D positions corresponding to the $i$-th points $\mathbf{p}_{i}$ and $\mathbf{q}_{i}$, respectively. As is shown in Fig. 3, we next consider circumferential lengths between two adjacent points defined by

$$
\begin{array}{lll}
\delta_{1}=\left\|\mathbf{r}_{1}-\mathbf{s}_{1}\right\|_{2}, & \delta_{2}=\left\|\mathbf{s}_{1}-\mathbf{t}_{1}\right\|_{2}, & \delta_{3}=\left\|\mathbf{t}_{1}-\mathbf{r}_{n}\right\|_{2}, \\
\delta_{4}=\left\|\mathbf{r}_{n}-\mathbf{s}_{n}\right\|_{2}, & \delta_{5}=\left\|\mathbf{s}_{n}-\mathbf{t}_{n}\right\|_{2}, & \delta_{6}=\left\|\mathbf{t}_{n}-\mathbf{r}_{1}\right\|_{2}, \\
\delta_{7}=\left\|\mathbf{r}_{2}-\mathbf{s}_{2}\right\|_{2}, & \delta_{8}=\left\|\mathbf{s}_{2}-\mathbf{t}_{2}\right\|_{2}, & \delta_{9}=\left\|\mathbf{t}_{2}-\mathbf{r}_{n-1}\right\|_{2}, \\
\delta_{10}=\left\|\mathbf{r}_{n-1}-\mathbf{s}_{n-1}\right\|_{2}, & \delta_{11}=\left\|\mathbf{s}_{n-1}-\mathbf{t}_{n-1}\right\|_{2}, & \delta_{12}=\left\|\mathbf{t}_{n-1}-\mathbf{r}_{2}\right\|_{2}, \\
\delta_{13}=\left\|\mathbf{r}_{3}-\mathbf{s}_{3}\right\|_{2}, & \delta_{14}=\left\|\mathbf{s}_{3}-\mathbf{t}_{3}\right\|_{2}, & \delta_{15}=\left\|\mathbf{t}_{3}-\mathbf{r}_{n-2}\right\|_{2}, \\
\delta_{16}=\left\|\mathbf{r}_{n-2}-\mathbf{s}_{n-2}\right\|_{2}, & \delta_{17}=\left\|\mathbf{s}_{n-2}-\mathbf{t}_{n-2}\right\|_{2}, & \delta_{18}=\left\|\mathbf{t}_{n-2}-\mathbf{r}_{3}\right\|_{2}, \\
\delta_{19}=\left\|\mathbf{r}_{m}-\mathbf{s}_{m}\right\|_{2}, & \delta_{20}=\left\|\mathbf{s}_{m}-\mathbf{t}_{m}\right\|_{2}, & \delta_{21}=\left\|\mathbf{t}_{m}-\mathbf{r}_{m}\right\|_{2},
\end{array}
$$

where $m=\operatorname{round}((n+1) / 2)$,

$$
\begin{aligned}
\mathbf{s}_{i}=\boldsymbol{\Psi} \mathbf{p}_{i}+\boldsymbol{\alpha}, & i=1, \ldots, n, \\
\mathbf{t}_{i}=\boldsymbol{\Phi} \mathbf{q}_{i}+\boldsymbol{\beta}, & i=1, \ldots, n,
\end{aligned}
$$

and the unknown matrices $\boldsymbol{\Psi}$ and $\boldsymbol{\Phi}$ transform 3D positions into contour points on their related apical view. The matrix $\Psi$ can be represented in the form

$$
\Psi=\left[\begin{array}{ccc}
\cos \varphi \cos \theta & -\cos \varphi \sin \theta & \sin \varphi \\
\sin \theta & \cos \theta & 0 \\
-\sin \varphi \cos \theta & \sin \varphi \sin \theta & \cos \varphi
\end{array}\right]
$$




$$
=\left[\begin{array}{ccc}
\cos \varphi & 0 & \sin \varphi \\
0 & 1 & 0 \\
-\sin \varphi & 0 & \cos \varphi
\end{array}\right]\left[\begin{array}{ccc}
\cos \theta & -\sin \theta & 0 \\
\sin \theta & \cos \theta & 0 \\
0 & 0 & 1
\end{array}\right],
$$

and $\boldsymbol{\Phi}$ has a similar representation. Hence, $\boldsymbol{\Psi}$ and $\boldsymbol{\Phi}$ satisfy the orthogonality conditions

$$
\boldsymbol{\Psi}^{T} \boldsymbol{\Psi}=\boldsymbol{\Psi} \boldsymbol{\Psi}^{T}=\mathbf{I}, \quad \boldsymbol{\Phi}^{T} \boldsymbol{\Phi}=\boldsymbol{\Phi} \boldsymbol{\Phi}^{T}=\mathbf{I},
$$

where $\mathbf{I}$ is the $3 \times 3$ identity matrix and $T$ is the operation of transposition.

We assume that the planes corresponding to the $\mathrm{A} 4 \mathrm{CH}, \mathrm{A} 3 \mathrm{CH}$ and $\mathrm{A} 2 \mathrm{CH}$ views are fixed throughout the entire cardiac cycle and consider the following model.

For given $\mathbf{p}_{i}, \mathbf{q}_{i}, \mathbf{r}_{i} \in \Omega \subseteq \mathbb{R}^{2} \times\{0\}, i=1, \ldots, n$, determine unknowns $\boldsymbol{\alpha}, \boldsymbol{\beta} \in[0,255]^{3}$ and $\Psi, \Phi \in \mathbb{R}^{3 \times 3}$ by minimising the function

$$
F(\boldsymbol{\alpha}, \boldsymbol{\beta}, \Psi, \Phi):=\frac{1}{7 \kappa} \sum_{\tau=\tau_{1}}^{\tau_{\kappa}} \sum_{j=1}^{21}\left(\delta_{j}^{\tau}\right)^{2},
$$

where $\kappa$ is the number of 2D echocardiography images acquired during the entire cardiac cycle and $\delta_{j}^{\tau}$ the value of $\delta_{j}, j=1, \ldots, 21$ at time $\tau$.

\subsection{Numerical scheme for finding $\alpha, \beta, \Psi$ and $\Phi$}

For a given initial guess $\boldsymbol{\alpha}^{(0)}, \boldsymbol{\beta}^{(0)}, \Psi^{(0)}$ and $\boldsymbol{\Phi}^{(0)}$, we update $\boldsymbol{\alpha}^{(k+1)}, \boldsymbol{\beta}^{(k+1)}, \Psi^{(k+1)}$ and $\boldsymbol{\Phi}^{(k+1)}$ sequentially $k=0,1, \ldots$ as follows:

$$
\begin{aligned}
& \boldsymbol{\alpha}^{(k+1)}:=\underset{\boldsymbol{\alpha} \in[-255,255]^{3}}{\operatorname{argmin}} F\left(\boldsymbol{\alpha}, \boldsymbol{\beta}^{(k)}, \boldsymbol{\Psi}^{(k)}, \boldsymbol{\Phi}^{(k)}\right)+\lambda_{\alpha}\left\|\boldsymbol{\alpha}-\boldsymbol{\alpha}^{(k)}\right\|_{2}^{2}, \\
& \boldsymbol{\beta}^{(k+1)}:=\underset{\boldsymbol{\beta} \in[-255,255]^{3}}{\operatorname{argmin}} F\left(\boldsymbol{\alpha}^{(k+1)}, \boldsymbol{\beta}, \boldsymbol{\Psi}^{(k)}, \boldsymbol{\Phi}^{(k)}\right)+\lambda_{\boldsymbol{\beta}}\left\|\boldsymbol{\beta}-\boldsymbol{\beta}^{(k)}\right\|_{2}^{2}, \\
& \boldsymbol{\Psi}^{(k+1)}:=\underset{\boldsymbol{\Psi}: \boldsymbol{\Psi}^{T} \boldsymbol{\Psi}=\boldsymbol{\Psi} \boldsymbol{\Psi}^{T}=\mathbf{I}}{\operatorname{argmin}} F\left(\boldsymbol{\alpha}^{(k+1)}, \boldsymbol{\beta}^{(k+1)}, \boldsymbol{\Psi}, \boldsymbol{\Phi}^{(k)}\right)+\lambda_{\psi}\left\|\boldsymbol{\Psi}-\boldsymbol{\Psi}^{(k)}\right\|_{F}^{2}, \\
& \boldsymbol{\Phi}^{(k+1)}:=\underset{\boldsymbol{\Phi}: \boldsymbol{\Phi}^{T} \boldsymbol{\Phi = \Phi} \boldsymbol{\Phi}^{T}=\mathbf{I}}{\operatorname{argmin}} F\left(\boldsymbol{\alpha}^{(k+1)}, \boldsymbol{\beta}^{(k+1)}, \boldsymbol{\Psi}^{(k+1)}, \boldsymbol{\Phi}\right)+\lambda_{\boldsymbol{\phi}}\left\|\boldsymbol{\Phi}-\boldsymbol{\Phi}^{(k)}\right\|_{F}^{2} .
\end{aligned}
$$

It follows from (2.5)-(2.6) that

$$
\begin{array}{ll}
\boldsymbol{\alpha}_{(j)}^{(k+1)}=\operatorname{median}\left\{-255, \tilde{\boldsymbol{\alpha}}_{(j)}^{(k)}, 255\right\}, & j=1,2,3, \\
\boldsymbol{\beta}_{(j)}^{(k+1)}=\operatorname{median}\left\{-255, \tilde{\boldsymbol{\beta}}_{(j)}^{(k)}, 255\right\}, & j=1,2,3,
\end{array}
$$

where $\boldsymbol{\alpha}_{(j)}$ denotes the $j$-th component of $\boldsymbol{\alpha}$ and

$$
\begin{aligned}
& \tilde{\boldsymbol{\alpha}}^{(k)}=\frac{1}{\lambda_{\alpha}+2}\left(\lambda_{\alpha} \boldsymbol{\alpha}^{(k)}+\boldsymbol{\beta}^{(k)}+\frac{1}{7 \kappa} \sum_{\tau=\tau_{1}}^{\tau_{\kappa}} \sum_{\substack{i=1,2,3, m, n-2, n-1, n}}\left(\mathbf{r}_{i}^{\tau}-2 \boldsymbol{\Psi}^{(k)} \mathbf{p}_{i}^{\tau}+\boldsymbol{\Phi}^{(k)} \mathbf{q}_{i}^{\tau}\right)\right), \\
& \tilde{\boldsymbol{\beta}}^{(k)}=\frac{1}{\lambda_{\beta}+2}\left(\lambda_{\beta} \boldsymbol{\beta}^{(k)}+\boldsymbol{\alpha}^{(k+1)}+\frac{1}{7 \kappa} \sum_{\substack{\tau=\tau_{1} \\
\tau_{k}}} \sum_{\substack{i=1,2,3, m, n-2, n-1, n}}\left(\mathbf{r}_{i}^{\tau}-2 \boldsymbol{\Phi}^{(k)} \mathbf{q}_{i}^{\tau}+\Psi^{(k)} \mathbf{p}_{i}^{\tau}\right)\right) .
\end{aligned}
$$


Writing the matrix

$$
\boldsymbol{\Psi}=\left[\begin{array}{lll}
\psi_{11} & \psi_{12} & \psi_{13} \\
\psi_{21} & \psi_{22} & \psi_{23} \\
\psi_{31} & \psi_{32} & \psi_{33}
\end{array}\right]
$$

as

$$
\begin{aligned}
& {\left[\begin{array}{lllllllll}
\psi_{11} & \psi_{12} & \psi_{13} & \psi_{21} & \psi_{22} & \psi_{23} & \psi_{31} & \psi_{32} & \psi_{33}
\end{array}\right]^{T} } \\
= & {\left[\begin{array}{lllllllll}
y_{1} & y_{2} & y_{3} & y_{4} & y_{5} & y_{6} & y_{7} & y_{8} & y_{9}
\end{array}\right]^{T} } \\
= & : \mathbf{y}
\end{aligned}
$$

and using the orthogonality conditions (2.3) yields

$$
\begin{aligned}
& \mathbf{y}^{T} \mathbf{N}_{k} \mathbf{y}=1, \quad k=1, \ldots, 6, \\
& \mathbf{y}^{T} \mathbf{N}_{k} \mathbf{y}=0, \\
& \mathbf{y}=\left[\begin{array}{lll}
y_{1} & \cdots & y_{9}
\end{array}\right]^{\top} \in \mathbb{R}^{9},
\end{aligned}
$$

where $\mathbf{N}_{1}, \ldots, \mathbf{N}_{12}$ are the following $9 \times 9$ matrices

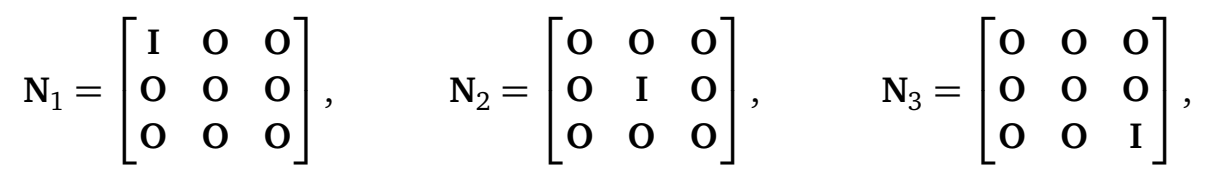

$$
\begin{aligned}
& \mathbf{N}_{7}=\left[\begin{array}{lll}
\mathbf{O} & \mathbf{I} & \mathbf{O} \\
\mathbf{I} & \mathbf{O} & \mathbf{O} \\
\mathbf{O} & \mathbf{O} & \mathbf{O}
\end{array}\right], \quad \mathbf{N}_{8}=\left[\begin{array}{ccc}
\mathbf{O} & \mathbf{O} & \mathbf{I} \\
\mathbf{O} & \mathbf{O} & \mathbf{O} \\
\mathbf{I} & \mathbf{O} & \mathbf{O}
\end{array}\right], \quad \mathbf{N}_{9}=\left[\begin{array}{lll}
\mathbf{O} & \mathbf{O} & \mathbf{O} \\
\mathbf{O} & \mathbf{O} & \mathbf{I} \\
\mathbf{O} & \mathbf{I} & \mathbf{O}
\end{array}\right] \\
& \mathbf{N}_{4}=\left[\begin{array}{ccc}
\mathbf{J}_{11} & \mathbf{0} & \mathbf{0} \\
\mathbf{O} & \mathbf{J}_{11} & \mathbf{0} \\
\mathbf{O} & \mathbf{O} & \mathbf{J}_{11}
\end{array}\right], \quad \mathbf{N}_{5}=\left[\begin{array}{ccc}
\mathbf{J}_{22} & \mathbf{0} & \mathbf{0} \\
\mathbf{O} & \mathbf{J}_{22} & \mathbf{0} \\
\mathbf{O} & \mathbf{O} & \mathbf{J}_{22}
\end{array}\right], \quad \mathbf{N}_{6}=\left[\begin{array}{ccc}
\mathbf{J}_{33} & \mathbf{O} & \mathbf{0} \\
\mathbf{O} & \mathbf{J}_{33} & \mathbf{0} \\
\mathbf{O} & \mathbf{O} & \mathbf{J}_{33}
\end{array}\right], \\
& \mathbf{N}_{10}=\left[\begin{array}{ccc}
\mathbf{J}_{12} & \mathbf{0} & \mathbf{0} \\
\mathbf{O} & \mathbf{J}_{12} & \mathbf{0} \\
\mathbf{O} & \mathbf{O} & \mathbf{J}_{12}
\end{array}\right], \quad \mathbf{N}_{11}=\left[\begin{array}{ccc}
\mathbf{J}_{13} & \mathbf{0} & \mathbf{0} \\
\mathbf{O} & \mathbf{J}_{13} & \mathbf{O} \\
\mathbf{O} & \mathbf{O} & \mathbf{J}_{13}
\end{array}\right], \quad \mathbf{N}_{12}=\left[\begin{array}{ccc}
\mathbf{J}_{23} & \mathbf{O} & \mathbf{0} \\
\mathbf{O} & \mathbf{J}_{23} & \mathbf{0} \\
\mathbf{O} & \mathbf{O} & \mathbf{J}_{23}
\end{array}\right] \text {. }
\end{aligned}
$$

Here, $\mathbf{J}_{i j}$ are the $3 \times 3$ symmetric matrices with 1 as $(i, j)$ and $(j, i)$-component and 0 otherwise. The problem (2.7) can be formulated as the following QCQP problem:

$$
\min _{\mathbf{y} \in \mathbb{R}^{9}} \mathbf{y}^{T}\left(\mathbf{H}_{1}+\lambda_{\psi} \mathbf{I}\right) \mathbf{y}-\left(\mathbf{f}^{T}+\lambda_{\psi}\left(\mathbf{y}^{(k-1)}\right)^{T}\right) \mathbf{y}
$$

subject to Eqs. (2.11),

where $\mathbf{H}_{1}$ is the $9 \times 9$ matrix

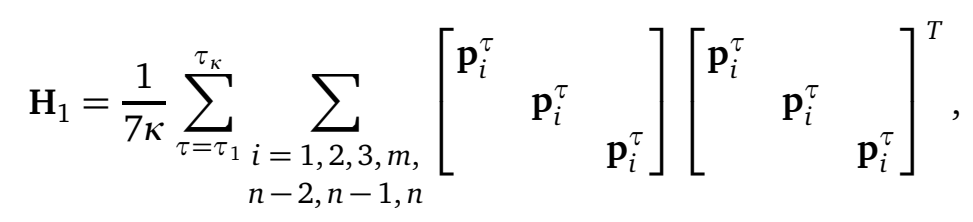


and $\mathbf{f}$ the $9 \times 1$ matrix

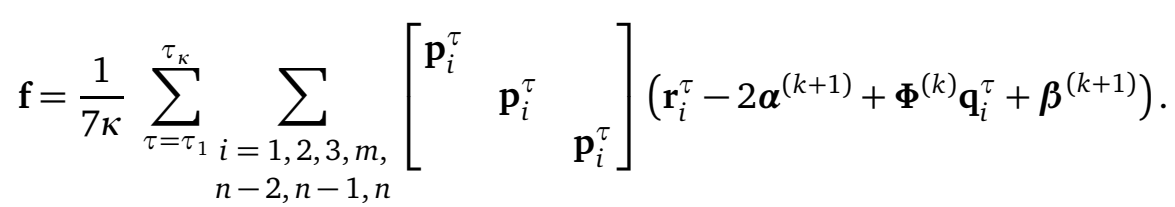

Analogously, in order to update the matrix

$$
\boldsymbol{\Phi}=\left[\begin{array}{lll}
\phi_{11} & \phi_{12} & \phi_{13} \\
\phi_{21} & \phi_{22} & \phi_{23} \\
\phi_{31} & \phi_{32} & \phi_{33}
\end{array}\right]
$$

we can reformulate the problem (2.8) as

$$
\begin{aligned}
& \min _{\mathbf{y} \in \mathbb{R}^{9}} \mathbf{y}^{T}\left(\mathbf{H}_{2}+\lambda_{\phi} \mathbf{I}\right) \mathbf{y}-\left(\mathbf{g}^{T}+\lambda_{\phi}\left(\mathbf{y}^{(k-1)}\right)^{T}\right) \mathbf{y} \\
& \quad \text { subject to Eqs. (2.11), }
\end{aligned}
$$

where $\mathbf{H}_{2}$ is the $9 \times 9$ matrix

$$
\mathbf{H}_{2}=\frac{1}{7 \kappa} \sum_{\substack{\tau=\tau_{1} \\
\tau_{\kappa}}} \sum_{\substack{i=2,3, m, n-2, n-1, n}}\left[\begin{array}{lll}
\mathbf{q}_{i}^{\tau} & & \\
& \mathbf{q}_{i}^{\tau} & \\
& & \mathbf{q}_{i}^{\tau}
\end{array}\right]\left[\begin{array}{lll}
\mathbf{q}_{i}^{\tau} & & \\
& \mathbf{q}_{i}^{\tau} & \\
& & \mathbf{q}_{i}^{\tau}
\end{array}\right]^{T}
$$

and $\mathbf{g}$ is the $9 \times 1$ matrix

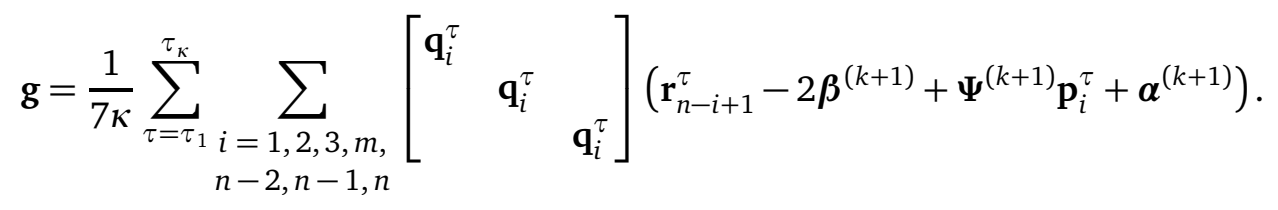

Since (2.12) and (2.13) are quadratically constrained quadratic programs, we can update $\Psi$ and $\Phi$ by using a QCQP solver.

At each iteration, we update one of the variables $\boldsymbol{\alpha}, \boldsymbol{\beta}, \boldsymbol{\Psi}, \boldsymbol{\Phi}$ with all others fixed. This numerical scheme is a proximal block coordinate descent method with cyclic order. The following theorem establishes the convergence of the proposed numerical scheme called PBCDC and applies to the following unconstrained optimisation reformulation of (2.4):

$$
\min _{\boldsymbol{\alpha}, \boldsymbol{\beta}, \Psi, \boldsymbol{\Phi}} \hat{F}(\boldsymbol{\alpha}, \boldsymbol{\beta}, \Psi, \boldsymbol{\Psi}):=\frac{1}{7 \kappa} \sum_{\tau=\tau_{1}}^{\tau_{\kappa}} \sum_{j=1}^{21}\left(\delta_{j}^{t}\right)^{2}+\iota_{U_{1}}(\boldsymbol{\Psi})+\iota_{U_{2}}(\boldsymbol{\Phi}),
$$

where $\iota_{U_{1}}(\boldsymbol{\Psi})$ is an indicator function of $U_{1}=\left\{\boldsymbol{\Psi}: \boldsymbol{\Psi}^{T} \boldsymbol{\Psi}=\boldsymbol{\Psi} \boldsymbol{\Psi}^{T}=\mathbf{I}\right\}$, i.e.

$$
\iota_{U_{1}}(\Psi)= \begin{cases}0, & \text { if } \Psi \in U_{1}, \\ \infty, & \text { otherwise, }\end{cases}
$$

and $\iota_{U_{2}}(\boldsymbol{\Phi})$ is an indicator function of $U_{2}=\left\{\boldsymbol{\Phi}: \boldsymbol{\Phi}^{T} \boldsymbol{\Phi}=\boldsymbol{\Phi} \boldsymbol{\Phi}^{T}=\mathbf{I}\right\}$. 
Theorem 2.1. Let $\left\{\boldsymbol{\alpha}^{(k)}, \boldsymbol{\beta}^{(k)}, \Psi^{(k)}, \Phi^{(k)}\right\}$ be a sequence generated by PBCDC. Then it converges to a critical point of (2.14).

The proof of this result is given in Appendix.

\section{Experimental Results}

We perform numerical experiments to evaluate the proposed model. Fig. 4 shows the process of experiments. First, synthetic data of varying LV is generated from real 3D LV volume data acquired by a SIEMENS ACUSON SC2000 imaging system with a 4Z1c probecf. Fig. 4(a). The cardiac cycle is divided into six time steps $\tau=\tau_{0}, \tau_{1}, \ldots, \tau_{6}$ of contraction and expansion. The LV boundary data extracted from the original 3D LV volume data are considered to be measured at $\tau=\tau_{0}$ and variable LV borders are generated to correspond to the time $\tau=\tau_{1}, \ldots, \tau_{6}$. It is assumed that the $\mathrm{LV}$ volume is contracted and expanded by applying myocardial strain of $25 \%$, three fixed planes are placed inside the LV and the LV borders are projected on the planes as shown in Fig. 4(b). After that, the projected 2D LV borders are used as an input to the proposed model $-\mathrm{cf}$. Fig. 4(c).

In the model implementation, the parameters of $\lambda_{\alpha}=0.1, \lambda_{\beta}=0.1, \lambda_{\psi}=100$ and $\lambda_{\phi}=100$ are used. We set the maximum number of iterations as 100 since it is observed that the relative errors of the estimated variables $\hat{\boldsymbol{\alpha}}, \hat{\boldsymbol{\beta}}, \hat{\boldsymbol{\Psi}}$ and $\hat{\boldsymbol{\Phi}}$ are rapidly reduced until around 100 iterations as shown in Fig. 5. The execution time of PBCDC in each iteration is about $11.12 \mathrm{sec}$. Note that the running time for QCQP takes up the most of the execution time. For $k \geq 100$, each relative error is less than a certain value as below

$$
\frac{\left\|\boldsymbol{\alpha}^{(k+1)}-\boldsymbol{\alpha}^{(k)}\right\|}{\left\|\boldsymbol{\alpha}^{(k)}\right\|}<0.0012, \quad \frac{\left\|\boldsymbol{\beta}^{(k+1)}-\boldsymbol{\beta}^{(k)}\right\|}{\left\|\boldsymbol{\beta}^{(k)}\right\|}<0.0009
$$

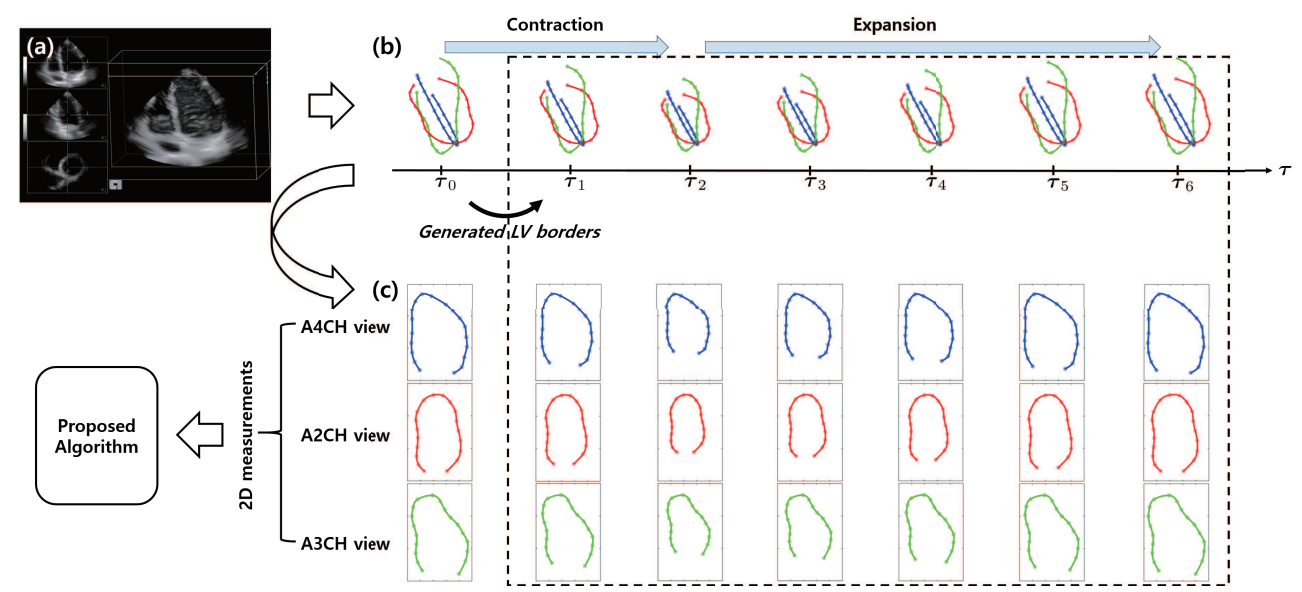

Figure 4: Experiment on synthetic data generated from real 3D LV volume data. (a) 3D volume data, (b) generated varying $\mathrm{LV}$ borders on $\mathrm{A} 4 \mathrm{CH}, \mathrm{A} 2 \mathrm{CH}$ and $\mathrm{A} 3 \mathrm{CH}$ views, (c) $2 \mathrm{D}$ LV borders as an input to the proposed model. 

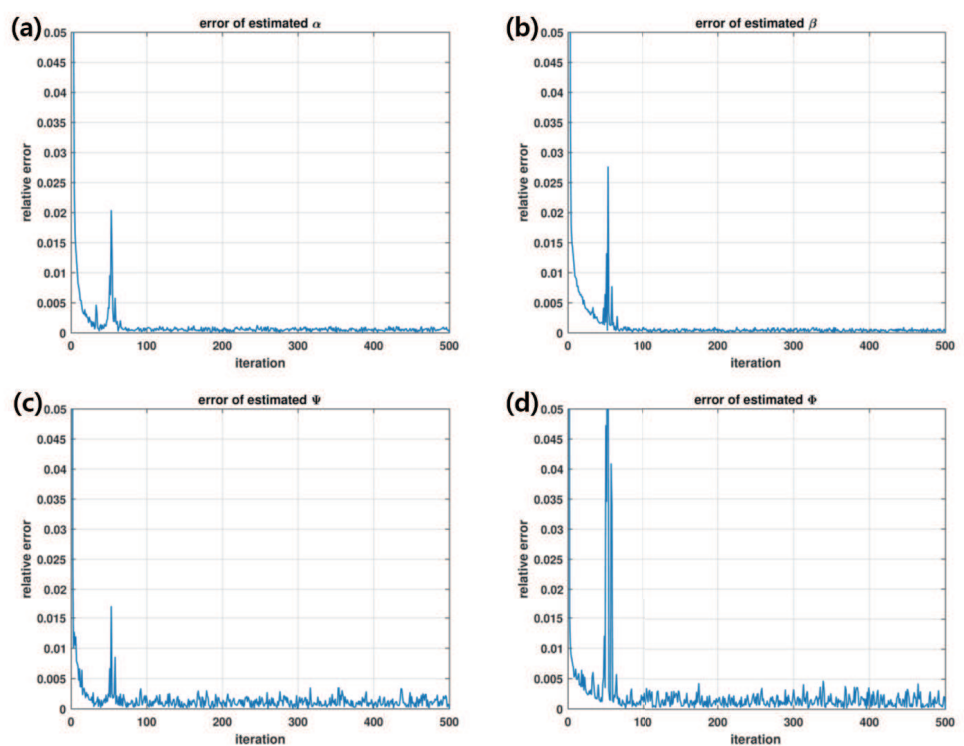

Figure 5: Relative errors of $\hat{\boldsymbol{\alpha}}, \hat{\boldsymbol{\beta}}, \hat{\boldsymbol{\Psi}}$ and $\hat{\boldsymbol{\Phi}}$ updated by the proposed algorithm depending on iterations.

$$
\frac{\left\|\Psi^{(k+1)}-\Psi^{(k)}\right\|}{\left\|\Psi^{(k)}\right\|}<0.0034, \quad \frac{\left\|\Phi^{(k+1)}-\Phi^{(k)}\right\|}{\left\|\Phi^{(k)}\right\|}<0.0047
$$

The values for variables $\boldsymbol{\alpha}, \boldsymbol{\beta}, \boldsymbol{\Psi}$ and $\boldsymbol{\Phi}$ are estimated as

$$
\begin{array}{ll}
\hat{\boldsymbol{\alpha}}=\left[\begin{array}{c}
25.48 \\
2.45 \\
54.83
\end{array}\right], & \hat{\Psi}=\left[\begin{array}{ccc}
0.5129 & 0.0723 & -0.8554 \\
-0.0350 & 0.9974 & 0.0634 \\
-0.8578 & 0.0026 & -0.5140
\end{array}\right], \\
\hat{\boldsymbol{\beta}}=\left[\begin{array}{c}
40.35 \\
-4.66 \\
-54.93
\end{array}\right], & \hat{\boldsymbol{\Phi}}=\left[\begin{array}{ccc}
0.3096 & 0.0431 & 0.9499 \\
0.0759 & 0.9947 & -0.0699 \\
0.9478 & -0.0937 & -0.3046
\end{array}\right] .
\end{array}
$$

By applying these values to (2.2), we obtain the spatial location of 2D LV borders used as an input to the model. Fig. 6(a) demonstrates estimating 3D position of 2D borders by using PBCDC. We observe how accurately the spatial locations of $\mathrm{A} 4 \mathrm{CH}$ and $\mathrm{A} 3 \mathrm{CH}$ views are restored for the $\mathrm{A} 2 \mathrm{CH}$ view. The difference between original and estimated $\mathrm{LV}$ control points $\mathrm{LV}$ on the $\mathrm{A} 4 \mathrm{CH}$ and $\mathrm{A} 3 \mathrm{CH}$ is computed by $l_{2}$-norm. The $\mathrm{LV}$ points on the $\mathrm{A} 4 \mathrm{CH}$ are restored with a relative error of about $10 \%$, while the A3CH LV points show the higher relative error of about $15 \%$. The errors of the restored $\mathrm{LV}$ control points at each step are listed in Table 1.

This paper focuses on the computation of 3D positions of the border points and does not consider the representation of 3D LV surfaces. Therefore, we use a free and opensource parametric 3D CAD modeler FreeCAD to represent the reconstructed 3D surface cf. Fig. 6(b). 


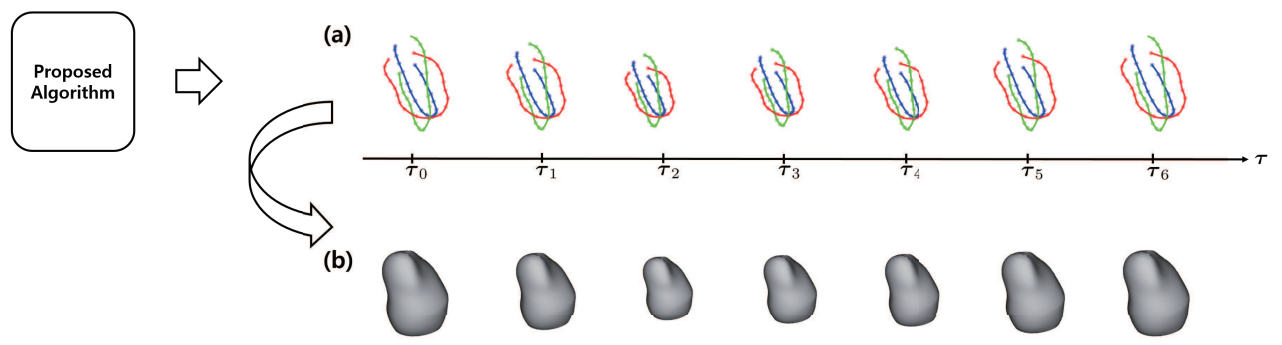

Figure 6: Experimental results. (a) Reconstructed $3 \mathrm{D} \mathrm{LV}$ borders on $\mathrm{A} 4 \mathrm{CH}, \mathrm{A} 2 \mathrm{CH}$ and $\mathrm{A} 3 \mathrm{CH}$ views, (b) Corresponding 3D LV surfaces.

All numerical tests are implemented on a $3.3 \mathrm{GHz}$ Core i9-7900X CPU with 128GB RAM, running on Windows 10 and MATLAB (Version 9.6).

Table 1: The errors of restored LV control points at contraction and expansion steps.

\begin{tabular}{|c|c|c|c|c|c|c|c|c|}
\hline \multicolumn{2}{|r|}{$\tau$} & $\tau_{1}$ & $\tau_{2}$ & $\tau_{3}$ & $\tau_{4}$ & $\tau_{5}$ & $\tau_{6}$ & mean \\
\hline \multirow[t]{2}{*}{$\mathrm{A} 4 \mathrm{CH}$} & $\|\mathbf{x}-\hat{\mathbf{x}}\|_{2}$ (pixels) & 27.65 & 24.21 & 25.30 & 26.45 & 28.89 & 30.17 & 27.11 \\
\hline & $\frac{\|\mathbf{x}-\hat{\mathbf{x}}\|_{2}}{\|\mathbf{x}\|_{2}} \quad$ (\%) & 9.89 & 10.39 & 10.18 & 10.01 & 9.79 & 9.71 & 10.00 \\
\hline \multirow[t]{2}{*}{$\mathrm{A} 3 \mathrm{CH}$} & $\|\mathbf{x}-\hat{\mathbf{x}}\|_{2}$ (pixels) & 41.26 & 34.61 & 36.82 & 39.04 & 43.48 & 45.70 & 40.15 \\
\hline & $\frac{\|\mathrm{x}-\hat{\mathbf{x}}\|_{2}}{\|\mathrm{x}\|_{2}}$ & 15.12 & 15.22 & 15.18 & 15.15 & 15.10 & 15.07 & 15.14 \\
\hline
\end{tabular}

\section{Conclusion}

We consider the recovery of 3D LV by using only 2D echocardiography data acquired in multiple views. The previously used models are formulated as non-convex constrained minimisation problems, which determine the 3D positions corresponding to 2D measured border points. It is assumed that the imaging planes corresponding to 3D view intersect at the same axis and the circumferential length of mitral annulus with small variation throughout the whole cardiac cycle is in the fixed range. However, the latter condition can be not verified by $2 \mathrm{D}$ imaging systems. Therefore, we propose a new model, which does not require the assumptions of $[1,2]$ but uses $2 \mathrm{D}$ echocardiography data acquired during the entire cardiac cycle. Assuming that the spatial shape of LV is not distorted in the process of contraction and expansion, we employ a nonconvex constrained minimisation problem. This model is a QCQP with respect to one of four variables with the others fixed. Therefore, we consider PBCDC using a QCQP solver [12] to exploit this structure. In unconstrained formulations of the model, the objective is a Kurdyka-Lojasiewicz function. This property allows to prove that the whole sequence generated by PBCDC applied to the model converges to a stationary point.

The model performs well in numerical experiments, which show that the model is mostly suitable. It can be of interest because there is no reliance on unpractical assumptions of other models. Nevertheless, the method requires further improvements, especially 
in terms of its accuracy. Thus the deviation higher than $10 \%$ between restored and original spatial positions are observed for the LV borders. This happens because the rotation of the LV border measured in each imaging plane is not considered. At the present, 3D echocardiography images have unsatisfactory spatial resolution. It needs further technological developments up to the level of 2D echocardiography imaging in terms of spatial and temporal resolution. To the best of our knowledge, the approach proposed is the first attempt to do it. Hence, there is no conventional model directly comparable to the model considered here. On the other hand, a recently developed automated 3D echocardiography chamber quantification technique measures LV volumes within $10 \%$ intermeasurement variability and this is considered as a performance closer to the real situation [13]. Therefore, although the reconstruction error in our model is not satisfactory, it is comparable to other results.

It should be also noted that the execution speed of the numerical algorithm should be improved. The total running time for calculation of the spatial position of LV borders moving in 6 steps is about 18.5 minutes, or about 3 minutes per step. In order to be practically valuable, the running time should be improved to a few seconds per step.

\section{Appendix A}

\section{A.1 Proof of Theorem 2.1}

First of all, we define semi-algebraic functions and the Kurdyka-Lojasiewicz property [5].

Definition A.1. A subset $S$ of $\mathbb{R}^{n}$ is a real semi-algebraic set if there exists a finite number of real polynomial functions $P_{i j}, Q_{i j}: \mathbb{R}^{n} \rightarrow \mathbb{R}$ such that

$$
S=\bigcup_{j=1}^{p} \bigcap_{i=1}^{q}\left\{x \in \mathbb{R}^{n}: P_{i j}(x)=0, Q_{i j}(x)<0\right\} .
$$

Furthermore, a function $\hat{F}: \mathbb{R}^{n} \rightarrow \mathbb{R} \cup\{+\infty\}$ is called semi-algebraic if its graph

$$
G(f):=\left\{(x, v) \in \mathbb{R}^{n+1}: \hat{F}(x)=v\right\}
$$

is a semi-algebraic subset of $\mathbb{R}^{n+1}$.

Definition A.2. The function $\hat{F}: \mathbb{R}^{n} \rightarrow \mathbb{R} \cup\{+\infty\}$ is said to have the Kurdyka-Lojasiewicz property at $\mathbf{x}^{*} \in \operatorname{dom} \partial \hat{F}$ if there exist $\mu \in(0,+\infty]$, a neighborhood $V$ of $\mathbf{x}^{*}$ and a continuous concave function $\gamma:[0, \mu) \rightarrow \mathbb{R}_{+}$such that,

1. $\gamma(0)=0$,

2. $\gamma$ is $C^{1}$ on $(0, \mu)$,

3. for all $\xi \in(0, \mu), \gamma^{\prime}(\xi)>0$, 
4. for all $\mathbf{x}$ in $V \cap\left[\hat{F}\left(\mathbf{x}^{*}\right)<\hat{F}(\mathbf{x})<\hat{F}\left(\mathbf{x}^{*}\right)+\mu\right]$, the Kurdyka-Lojasiewicz inequality holds

$$
\gamma^{\prime}\left(\hat{F}(\mathbf{x})-\hat{F}\left(\mathbf{x}^{*}\right)\right) \operatorname{dist}(0, \partial \hat{F}(\mathbf{x})) \geq 1 \text {. }
$$

Proper lower semicontinuous functions which satisfy the Kurdyka-Lojasiewicz inequality at each point of $\partial \hat{F}$ are called KL functions.

The proof of Theorem 2.1 can be obtained by applying the following result.

Theorem A.1 (cf. Attouch et al. [5, Theorem 6.2]). Let

$$
\hat{F}(\mathbf{x}): \mathbb{R}^{n_{1}} \times \cdots \times \mathbb{R}^{n_{p}} \rightarrow \mathbb{R} \cup\{+\infty\}=Q\left(\mathbf{x}_{1}, \ldots, \mathbf{x}_{p}\right)+\sum_{i=1}^{p} f_{i}\left(\mathbf{x}_{i}\right),
$$

where $Q$ is a $C^{1}$ function with locally Lipschitz continuous gradient, and $f_{i}: \mathbb{R}^{n_{i}} \rightarrow \mathbb{R} \cup\{+\infty\}$, $i=1, \ldots, p$ are proper lower semicontinuous $K L$ functions bounded from below. If the sequence $\left\{\mathbf{x}^{k}\right\}_{k \in N}$ is bounded and satisfies the conditions:

(C1) (Sufficient decrease condition) For each $k \geq 0$, there exists a positive constant $\lambda$ such that

$$
\hat{F}\left(\mathbf{x}^{k+1}\right)+\lambda\left\|\mathbf{x}^{k+1}-\mathbf{x}^{k}\right\|^{2} \leq \hat{F}\left(\mathbf{x}^{k}\right) .
$$

(C2) (Relative error condition) For each $k \geq 0$, there exists a positive constant $M$ and $a \mathbf{w}^{k+1} \in$ $\partial \hat{F}\left(\mathbf{x}^{k+1}\right)$ such that, for all $k$,

$$
\left\|\mathbf{w}^{k+1}\right\| \leq M\left\|\mathbf{x}^{k+1}-\mathbf{x}^{k}\right\| .
$$

(C3) (Continuity condition) There exists a subsequence $\left\{\mathbf{x}^{k_{j}}\right\}_{j \in N}$ and $\tilde{\mathbf{x}}$ such that

$$
\mathbf{x}^{k_{j}} \rightarrow \tilde{\mathbf{x}} \text { and } \hat{F}\left(\mathbf{x}^{k_{j}}\right) \rightarrow \hat{F}(\tilde{\mathbf{x}}), \quad j \rightarrow \infty
$$

then it converges to a critical point $\overline{\mathbf{x}}$ of $\hat{F}$. Moreover the sequence $\left\{\mathbf{x}^{k}\right\}_{k \in N}$ has a finite length -i.e.

$$
\sum_{k=0}^{\infty}\left\|\mathbf{x}^{k+1}-\mathbf{x}^{k}\right\|<+\infty
$$

The sequences $\left\{\boldsymbol{\alpha}^{(k)}, \boldsymbol{\beta}^{(k)}, \Psi^{(k)}, \Phi^{(k)}\right\}$ are bounded because of the boundedness of the variables $\boldsymbol{\alpha}, \boldsymbol{\beta}$ and the constraints (2.11) for $\boldsymbol{\Psi}$ and $\boldsymbol{\Phi}$. The objective function $\hat{F}$ of (2.14) is continuous on its domain. Therefore, in order to prove Theorem 2.1 it suffices to show that the sequence generated by PBCDC satisfies conditions (C1), (C2) and $\hat{F}$ satisfies the KL property.

Assume that conditions (C1) and (C2) are satisfied, and define the subdifferential $\partial \hat{F}(x)$ of $\hat{F}$ at $x \in \operatorname{dom} \hat{F}$ by

$$
\partial \hat{F}(x):=\left\{x^{*} \mathbb{R}^{n}: \exists x_{n} \rightarrow x, \hat{F}\left(x_{n}\right) \rightarrow \hat{F}(x), x_{n}^{*} \in \hat{\partial} \hat{F}\left(x_{n}\right) \rightarrow x^{*}\right\},
$$


where $\hat{\partial} \hat{F}(x)$ is the Fréchet subdifferential of $\hat{F}$ at $x$ and

$$
\lim \inf _{y \neq x, y \rightarrow x} \frac{1}{\|x-y\|}\left[\hat{F}(y)-\hat{F}(x)-\left\langle x^{*}, y-x\right\rangle\right] \geq 0 .
$$

Let $\mathbf{x}=\left(\mathbf{x}_{1}, \mathbf{x}_{2}, \mathbf{x}_{3}, \mathbf{x}_{4}\right)$ with $\mathbf{x}_{1}=\boldsymbol{\alpha}, \mathbf{x}_{2}=\boldsymbol{\beta}, \mathbf{x}_{3}=\boldsymbol{\Psi}, \mathbf{x}_{4}=\boldsymbol{\Phi}$. By the numerical scheme PBCDC - i.e. by iterations (2.5)-(2.8), for all $k \geq 0$ we have

$$
\hat{F}\left(\mathbf{x}^{k+1}\right)+\lambda\left\|\mathbf{x}^{k+1}-\mathbf{x}^{k}\right\|^{2} \leq \hat{F}\left(\mathbf{x}^{k}\right),
$$

where

$$
\left\|\mathbf{x}^{k+1}-\mathbf{x}^{k}\right\|=\sqrt{\sum_{i=1}^{2}\left\|\mathbf{x}_{i}^{k+1}-\mathbf{x}_{i}^{k}\right\|+\sum_{i=3}^{4}\left\|\mathbf{x}_{i}^{k+1}-\mathbf{x}_{i}^{k}\right\|_{F}^{2}}
$$

and $\lambda=\min \left\{\lambda_{\alpha}, \lambda_{\beta}, \lambda_{\psi}, \lambda_{\Phi}\right\}$. Thus, condition (C1) is satisfied.

The optimality condition for each of the problems in (2.5)-(2.8) gives

$$
\begin{aligned}
& \nabla_{\mathbf{x}_{1}} F\left(\mathbf{x}_{1}^{k+1}, \mathbf{x}_{2}^{k}, \mathbf{x}_{3}^{k}, \mathbf{x}_{4}^{k}\right)+\lambda_{\alpha}\left(\mathbf{x}_{1}^{k+1}-\mathbf{x}_{1}^{k}\right)=0, \\
& \nabla_{\mathbf{x}_{2}} F\left(\mathbf{x}_{1}^{k+1}, \mathbf{x}_{2}^{k+1}, \mathbf{x}_{3}^{k}, \mathbf{x}_{4}^{k}\right)+\lambda_{\beta}\left(\mathbf{x}_{2}^{k+1}-\mathbf{x}_{2}^{k}\right)=0, \\
& \nabla_{\mathbf{x}_{3}} F\left(\mathbf{x}_{1}^{k+1}, \mathbf{x}_{2}^{k+1}, \mathbf{x}_{3}^{k+1}, \mathbf{x}_{4}^{k}\right)+\lambda_{\psi}\left(\mathbf{x}_{3}^{k+1}-\mathbf{x}_{3}^{k}\right)+v_{\psi}=0, \\
& \nabla_{\mathbf{x}_{4}} F\left(\mathbf{x}_{1}^{k+1}, \mathbf{x}_{2}^{k+1}, \mathbf{x}_{3}^{k+1}, \mathbf{x}_{4}^{k+1}\right)+\lambda_{\Phi}\left(\mathbf{x}_{4}^{k+1}-\mathbf{x}_{4}^{k}\right)+v_{\Phi}=0
\end{aligned}
$$

for some $v_{\psi} \in \partial \iota_{U_{1}}\left(\mathbf{x}_{3}^{k+1}\right)$ and $v_{\Phi} \in \partial \iota_{U_{2}}\left(\mathbf{x}_{4}^{k+1}\right)$. Since the objective functions in (2.5)-(2.8) are quadratic, the equations in (A.1) yield the existence of a $\mathbf{w}^{k+1} \in \partial \hat{F}\left(\mathbf{x}^{k+1}\right)$ such that, for all $k$,

$$
\left\|\mathbf{w}^{k+1}\right\| \leq M\left\|\mathbf{x}^{k+1}-\mathbf{x}^{k}\right\| \text { for some } \quad M>0 .
$$

Thus the condition (C2) is also satisfied.

Next, we show that the function $\hat{F}$ has the Kurdyka-Lojasiewicz property. The orthogonality condition (2.3) of $\boldsymbol{\Psi}$ is expressed as the Eqs. (2.11). So is that of $\boldsymbol{\Phi}$. The equations in (2.11) are polynomial equations. Hence the constraint set for the orthogonality condition is a semi-algebraic set [7]. Indicator functions of semi-algebraic sets are semi-algebraic functions [4]. By the definition of $\delta_{i}, i=1, \ldots, 21$ in (2.1), the objective function of (2.4) is polynomial and hence is a semi-algebraic function. Finally, the objective function $\hat{F}$ is a sum of semi-algebraic functions and hence it is a semi-algebraic function [7]. Then $\hat{F}$ satisfies the Kurdyka-Lojasiewicz property [8].

\section{Acknowledgments}

The first author was supported by the National Institute for Mathematical Sciences (Grant No. 20910000) and by the National Research Foundation of Korea funded by the Korea government (Grant Nos. 2017R1E1A1A03070382, 2021R1A2C1010993). The second author was supported by the National Research Foundation of Korea (Grant Nos. 2016R1A5A1008055, 2019R1F1A1057051). 


\section{References}

[1] C.Y. Ahn and S. Yun, A study on the 3D position estimation of ventricular borders extracted from 2D echocardiography data, Comput. Math. Appl. 75, 1143-1158 (2018).

[2] C.Y. Ahn and S. Yun, A mathematical model for the 3D location estimation of $2 D$ echocardiography data, Appl. Math. Lett. 88, 186-192 (2019).

[3] G.S. Alberti, H. Ammari, F. Romero and T. Wintz, Mathematical analysis of ultrafast ultrasound imaging, SIAM J. Appl. Math. 77, 1-25 (2017).

[4] H. Attouch and J. Bolte, On the convergence of the proximal algorithm for nonsmooth functions involving analytic features, Math. Program. 116, 5-16 (2009).

[5] H. Attouch, J. Bolte and B.F. Svaiter, Convergence of descent methods for semi-algebraic and tame problems: proximal algorithms, forward-backward splitting, and regularized Gauss-Seidel methods, Math. Program. 137, 91-129 (2013).

[6] A.I. Aviles, T. Widlak, A. Casals, M.M. Nillesen and H. Ammari, Robust cardiac motion estimation using ultrafast ultrasound data: A low-rank-topology-preserving approach, Phys. Med. Biol. 62, 4831-4851 (2017).

[7] J. Bochnak, M. Coste and M.F. Roy, Real Algebraic Geometry 3, Springer-Verlag (1998).

[8] J. Bolte, S. Sabach and M. Teboulle, Proximal alternating linearized minimization for nonconvex and nonsmooth problems, Math. Program., 1-36 (2013).

[9] C. Bonciu, R. Weber and C. Leger, $4 D$ reconstruction of the left ventricle during a single heart beat from ultrasound imaging, Image Vision Comput. 19, 401-412 (2001).

[10] S. Boyd and L. Vandenberghe, Convex Optimization, Cambridge University Press (2004).

[11] G. Coppini, R. Poli, G. Valli, Recovery of the 3-D shape of the left ventricle from echocardiographic images, IEEE Trans. Med. Imaging 14, 301-317 (1995).

[12] J. Currie and D.I. Wilson, OPTI: Lowering the Barrier Between Open Source Optimizers and the Industrial MATLAB User, Foundations of Computer-Aided Process Operations (2012).

[13] R.M. Lang, K. Addetia, A. Narang and V. Mor-Avi, 3-dimensional echocardiography: Latest developments and future directions, JACC: Cardiovascular Imaging 11, 1854-1878 (2018).

[14] R. Lang, S.A. Goldstein, I. Kronzon, B.K. Khandheria and V. Mor-Avi, ASE's Comprehensive Echocardiography, Elsevier Health Sciences (2015).

[15] L. Mercier, T. Lang $\emptyset$, F. Lindseth and D.L. Collins, A review of calibration techniques for freehand 3-D ultrasound systems, Ultrasound Med. Biol. 31, 143-165 (2005).

[16] V. Mor-Avi, C. Jenkins, H.P. Kühl, H.J. Nesser, T. Marwick, A. Franke, C. Ebner, B.H. Freed, R. Steringer-Mascherbauer, H. Pollard, L. Weinert, J. Niel, L. Sugeng and R.M. Lang, Real-time 3-dimensional echocardiographic quantification of left ventricular volumes: multicenter study for validation with magnetic resonance imaging and investigation of sources of error, JACC Cardiovasc. Imaging. 1, 413-423 (2008).

[17] V. Mor-Avi, L. Sugeng and R.M. Lang, Real-time 3-dimensional echocardiography: an integral component of the routine echocardiographic examination in adult patients?, Circulation 119, 314-329 (2009).

[18] N. Parikh and S. Boyd, Proximal Algorithms, Foundations and Trends in Optimization 1, 123231 (2014).

[19] X. Ye, J.A. Noble and D. Atkinson, 3-D freehand echocardiography for automatic left ventricle reconstruction and analysis based on multiple acoustic windows, IEEE Trans. Med. Imaging 21, 1051-1058 (2002). 